\title{
Initial Trauma Database in a University Hospital in Malaysia
}

Affirul Chairil Ariffin¹, Mohamed Hajhamad², Firdaus Hayati³ ${ }^{*}$, Nornazirah Azizan4, Zamri Zuhdi², Azlanudin Azman², Ikhwan Sani Mohamad ${ }^{5}$, Hairol Othman², Razman Jarmin², Jae Gil Lee ${ }^{6}$

1 Department of Surgery,

Universiti Sains Islam Malaysia,

Kuala Lumpur, Malaysia

2 Department of Surgery,

Universiti Kebangsaan Malaysia,

Kuala Lumpur, Malaysia

${ }^{3}$ Department of Surgery, Faculty of Medicine and Health Sciences, Universiti Malaysia

Sabah, Kota Kinabalu, Sabah, Malaysia

${ }^{4}$ Department of Pathobiology and Medical

Diagnostic, Faculty of Medicine and Health

Sciences, Universiti Malaysia Sabah,

Kota Kinabalu,Sabah, Malaysia

${ }^{5}$ Department of Surgery, Universiti Sains

Malaysia, Kubang Kerian, Kelantan, Malaysia

${ }^{6}$ Department of Surgery, Yonsei University

College of Medicine, Seoul, South Korea

*Corresponding author's email:

firdaushayati@gmail.com

Received: 25 April 2018

Accepted: 11 June 2019

Keywords:

trauma, injuries trauma severity

index, data banks, registries

\section{ABSTRACT}

Trauma is a major health problem in Malaysia. An understanding of the trauma epidemiology is important in developing a reliable trauma service. The aim of this study is to understand the pattern of trauma in our institution and to highlight the need for a dedicated trauma service. In this database, 142 cases were included. Majority were males $(127,89.4 \%)$. Most common injury types are motor vehicle accidents $(87.3 \%)$ followed by falls $(7.7 \%)$ and stabs (3.5\%). Most Injury Severity Score (ISS) falls under moderate score with $38.7 \%$. Mean Abbreviated Injury Score (AIS) was 3 with most involving the chest and $90 \%$ of the patients have injuries involving at least 2 regions. Average hospital length of stay (LOS) was 11.4 days \pm 11.5 SD; with most patients $(\mathbf{7 1 . 8 \%})$ were discharged without permanent disability. The mortality rate was $9.2 \%$ with all having ISS $>16$. ISS found to be strongly related to longer hospital stay and worse outcome $(0.59, \mathrm{p}<\mathbf{0 . 0 0 0 1}, \mathbf{0 . 4}, \mathrm{p}<$ $0.0001)$. This data is equivalent to the compared registries from 4 different trauma centres. However, steps need to be taken to improve this database. In conclusion, this university hospital receives a reasonable load of trauma cases yearly which is equivalent with other trauma centres. The increasing trauma cases will benefit from an implementation of a dedicated trauma service. This trauma database needs more depth in its elements and better data handling to ensure a quality and complete registry.

\section{INTRODUCTION}

Trauma remains one of the major causes of death worldwide. It causes a significant morbidity and mortality especially among 
young adults. With the rise of urbanization and modernization, trauma cases are expected to rapidly increase. World Health Organization (WHO) has concluded that trauma is a cause for $11 \%$ mortality annually'. Countries with established trauma systems with designated trauma centres such as in United States, Australia and New Zealand have proved that a dedicated trauma organization and systems works well with positive effect in improving patients' outcome $e^{2,3,4}$.

However, in most developing countries such as Malaysia, we rely on the trauma team activation approach which consists of general surgeons, emergency physicians and other supportive departments. Although it is a reasonable strategy approaching trauma cases, but the efficiency seems decreasing. Issues such as delayed consultations and referrals, missed findings and unreasonable investigation request via phone calls are just a few. This is attributed to the time constraint of the onduty subspecialty physicians who must juggle between elective cases and clinic commitments. There is no analysis ever done looking at the outcome of this approach. We hope this article will show the needed evidence for a dedicated trauma services implementation.

In developing a trauma services and system, trauma registry is one of the major key components. The implementation of trauma registries in developed countries enables them to promote improvement of patient care, mortality and final outcomes ${ }^{5}$. It also plays a large role in prevention and quality assessment. However, articles on active trauma registries among developing countries are scarce and limited. Malaysia trauma system is in its nascent stage with no established national trauma database. The data collection and maintenance posed a challenged with limited trauma centres and limited funding of the registries. Other problem encountered during data collection is missing and incomplete data. This missing data presents a challenge when standardized registries are required as a benchmark. The objective of this study is to share preliminary trauma data collected in our university hospital and identify the weakness with optimism to improve for future collection.

\section{MATERIALS AND METHODS}

This study is a retrospective data collection from 2011 - 2013. The collection of trauma data was performed in Universiti Kebangsaan Malaysia Medical Centre (UKMMC), one of the university hospitals that serves as a tertiary centre in Kuala Lumpur, Malaysia. The figures were amassed from the trauma census kept by surgery, emergency and trauma department and intensive care unit. It consists of polytrauma patients and trauma cases presented under general surgery unit only. Pure neurosurgery and orthopaedic trauma cases were not included in the analysis due to incomplete and missing data.

The data was collected in a modified excel sheet with designated elements. Data elements collected include the demographics (gender, age, ethnic, and trauma type), time and day of injury and hospital presentation. Other parameters include the Injury Severity Score (ISS), management provided, the length of stay, the survival and difference in weekdays and weekends admissions and outcome. The Injury Severity Score (ISS) was calculated manually based on the Abbreviated Injury Scale (AIS) values ${ }^{6,7}$. The data was calculated using mean and percentages. Pearson's correlation was used to determine the association between the Injury Severity Score (ISS), length of stay and outcomes.

\section{RESULTS}

There were 142 patients presented to our institution in recorded year (Table 1). Males were predominant with $89.4 \%$. Trauma cases were common among the two major ethnics in Malaysia, Malay and Chinese with $41.5 \%$ and $39.4 \%$ respectively. The majority proportion of injury occurs within 15 to 59-year-old with $86.7 \%$ while the remaining patients occurs in 60 to 74-year-old and more than 74-year-old with each $7.1 \%$ and $5.6 \%$ respectively. We only see one paediatric trauma in our registry. 
Table 1 Demographic data $(n=142)$

\begin{tabular}{|l|l|c|}
\hline \multicolumn{2}{|l|}{ Variables } & Frequency (\%) \\
\hline Age group (years) & $1(0.6)$ \\
\hline & $0-14$ & $123(86.7)$ \\
\hline & $15-59$ & $10(7.1)$ \\
\hline & $60-74$ & $8(5.6)$ \\
\hline \multicolumn{2}{|l|}{$>74$} & $127(89.4)$ \\
\hline Gender & Male & $15(10.6)$ \\
\hline \multicolumn{2}{|l|}{ Female } & \\
\hline Ethnicity & $59(41.5)$ \\
\hline & Malay & $56(39.4)$ \\
\hline & Chinese & $14(9.9)$ \\
\hline & Indian & $13(9.2)$ \\
\hline & Others & \\
\hline
\end{tabular}

Motor vehicle accident (MVA) remains the major cause for trauma in our institution with $87.3 \%$ (Table 2). Other cause of trauma was fall with $7.7 \%$. Penetrating trauma was low in our data with $3.5 \%$ had stab wound and only one presented with gunshot wound. Presentation of trauma cases were more during the weekdays compared to weekends with $63.4 \%$ and $36.6 \%$ respectively. There was no difference in the time of presentation. Most patients present with more than one regional injury. There were total of 238 injuries for 142 patients with more than $90 \%$ have at least 2 different regions of injuries. The head and chest regions were the most involved with $23.5 \%$ and $33.1 \%$ respectively.

Table 2 Trauma type and presentation detail

\begin{tabular}{|c|c|c|}
\hline Variables & & Frequency (\%) \\
\hline \multicolumn{3}{|c|}{ Trauma type } \\
\hline & Motor vehicle accident & $124(87.3)$ \\
\hline & Fall & $11(7.7)$ \\
\hline & Stab injury & $5(3.5)$ \\
\hline & Gunshot & $1(0.7)$ \\
\hline & Assault & $1(0.7)$ \\
\hline \multicolumn{3}{|c|}{ Presentation day } \\
\hline & Weekdays & $90(63.4)$ \\
\hline & Weekends and holidays & $52(36.6)$ \\
\hline \multicolumn{3}{|c|}{ Presentation time } \\
\hline & Day (07:00 a.m. - 07:00 p.m.) & $77(54.2)$ \\
\hline & Night (07:00 p.m. - 07:00 a.m.) & $65(45.8)$ \\
\hline
\end{tabular}

The mean AIS score is 3 with the majority involving the chest. There were significant numbers of AIS score of more than 5 which mostly involving the head and neck region. The mean ISS score was 18. Most of the patients in the registry are designated under ISS $9-15$ (38.7\%) followed by ISS $\geq 16$ with $22.5 \%$. A total of $32.4 \%$ of patients fall under critical ISS $>25$ (Table 3). There were 10 deaths within the registry with all having ISS $\geq 16$. All 10 mortalities involved the head region. Patients undergoing major intervention had longer length of stay (19.3 days) compared to both in the conservative and minor intervention 
group ( 4.5 days and 7.8 days respectively). The statistical analysis using Pearson's correlation shows a strong association between ISS and length of stay $(0.40, p<0.0001,95 \% \mathrm{Cl} 0.25$ to
$0.53)$ and final outcomes $(0.59, p<0.0001,95 \%$ $\mathrm{Cl} 0.47$ to 0.69 ) (Table 4). The outcomes were shown to be better in patients with ISS $\leq 16$, in which ISS was inversely related to length of stay and final outcomes.

Table 3 Severity assessment, length of stay and outcomes

\begin{tabular}{|c|c|c|}
\hline Variables & & Frequency (\%) \\
\hline \multicolumn{3}{|c|}{ Injury severity score } \\
\hline & Minor $(1-8)$ & $9(6.3)$ \\
\hline & Moderate $(9-15)$ & $55(38.7)$ \\
\hline & Serious $(16-24)$ & $32(22.5)$ \\
\hline & Severe $(25-49)$ & $42(29.6)$ \\
\hline & Critical $(50-74)$ & $4(2.8)$ \\
\hline & Maximum (75) & $0(0)$ \\
\hline \multicolumn{3}{|c|}{ Management } \\
\hline & Conservative only & $45(31.7)$ \\
\hline & Minimal intervention & $38(26.8)$ \\
\hline & Major surgical intervention & $58(40.8)$ \\
\hline & Referral to other centre & $1(0.7)$ \\
\hline \multicolumn{2}{|c|}{ Length of stay } & Days \\
\hline & Conservative only & 4.5 \\
\hline & Minimal intervention & 7.8 \\
\hline & Major intervention & 19.3 \\
\hline \multicolumn{3}{|c|}{ Final outcome } \\
\hline & Discharge, no disability & $11(7.7)$ \\
\hline & Discharge, non-permanent disability & $102(71.8)$ \\
\hline & Discharge, permanent disability & $16(11.3)$ \\
\hline & Death & $13(9.2)$ \\
\hline
\end{tabular}

Table 4 Correlation between ISS, length of stay and final outcomes

\begin{tabular}{|l|c|c|c|c|}
\hline \multirow{2}{*}{ Variables } & \multirow{2}{*}{$p$-value } & $r^{*}$ & \multicolumn{2}{|c|}{$95 \% \mathrm{Cl}$} \\
\cline { 3 - 5 } & & & Lower & Upper \\
\hline Length of stay & $<0.0001$ & 0.59 & 0.47 & 0.69 \\
\hline Final outcomes & $<0.0001$ & 0.40 & 0.25 & 0.53 \\
\hline
\end{tabular}

*Pearson's correlation

\section{DISCUSSION}

Trauma system has been shown to significantly improve the mortality rates in most developed countries. The establishment of working trauma systems requires meticulous planning, massive funding, appropriate government support and stringent policies. Despite its necessity is being argued previously but it has proven to be effective and reliable ${ }^{6}$. However, not every country is able to have such designation due to the lack of trauma system, integration of emergency services and source of funding ${ }^{8}$.

The objective of this article is to share our trauma data in one of the tertiary university hospitals in Klang Valley, Malaysia. Our hospital is one of the 10 major hospitals in the city centre vicinity. Despite having the resources 
and facilities, we are still lacking a dedicated trauma services within our institution. This may be attributed due to the lack of interest in trauma care among specialist, absence of a trauma surgeons and no appropriate support and funding from the governing body. Trauma cases in our institution are currently managed by a two-tiered trauma team activation consists of experts from multiple disciplines such as emergency physician, general surgery and other specialty unit. The activation of specialty depends on the type of injuries presented. This system is implemented in most countries which are yet to have trauma centres. It has previously shown to be reliable and effective ${ }^{9}$. However, the protocol and implementation vary among centres with no standardized managements.

This does not mean a trauma team response is not without any difficulties. With the advance in technology and new management in treating complex cases, most of the time is dedicated to non-trauma and elective works. These surgeons have found difficulty to divide equally their time between their specialty cases on top of dealing with occasional trauma cases. As an alternative, a dedicated trauma services could be started to cater the increasing trauma patients. There is strong evidence to suggest the implementation of a dedicated trauma services improves the patients' outcome. Few studies have shown the effectiveness of a dedicated trauma surgeon and trauma and critical care services in improving the survival outcome among trauma patients ${ }^{10,11}$. More interestingly, a study done in Australia has showed small but significant reduction of $8 \%$ of mortality in severely injured patients ${ }^{12}$. We hope our data will emphasize the need for such services in our institution.

Another important concept in a trauma system is keeping a good trauma registry. This is important in quality improvement of trauma care $^{13}$. Developed countries with established trauma system has been using trauma registries for in-house quality control and directing government policies ${ }^{14}$. Nevertheless, to develop a trauma registry, few setbacks need to be considered. Issues such as data collection, appropriateness of the collected elements, the ability to monitor and funding are just a tip of an iceberg in its development ${ }^{15,16}$.

Our centre is still far from developing a proper registry. However, an alternative to this now is compilation of trauma data based on the hospital admission and medical records. Majority of the data was collected using elements from another proposed trauma data registry and keyed into a modified Microsoft excel sheet.

Our institute received a reasonable load of trauma cases per year although this data does not include pure neurosurgery and orthopaedic trauma. A traffic accident was the major cause of trauma cases in our data. A total of $55 \%$ of patients presented to us had ISS more than 15 . This signifies a high volume of cases with severe injuries. We compare ours with recent data from four trauma centres and their respective registries. Our data is equivalent with most of the registries in terms of cases presentation and severity assessment ${ }^{17,} 18,19$, ${ }^{20}$. The type of injuries encountered in most trauma centre is traffic accident except for Byun CS et al. (2015) whereby fall is predominant. However, our mortality is higher than Hasler RM et al. (2014) ${ }^{14}$ and Byun CS et al. (2015) ${ }^{21}$. On the other hand, our mortality is similar with the data from the trauma centre in Europe. Though now mortality alone cannot be the measure of a trauma system success but is it a simple method to evaluate using a basic trauma database such as our institution ${ }^{21}$.

Looking at the elements captured in the registries, we can conclude from the comparison table that most demographic data were similar. ISS is recorded consistently in all registries although the categorization was varied. However, elements used in the calculation Revised Trauma Score (RTS) and Glasgow Coma scale were not consistently recorded as per our database. This may be 
attributed to the different objective and priority of each registries data handlers. If we compare with established trauma registries such as National Trauma Databank, Germany trauma registry, Korean National Trauma Databank, Australia and New Zealand trauma data registries and UK Trauma Audit and Research Network (TARN), our databank is lacking significant elements which include vital signs, Glasgow Coma Scale (GCS), prehospital data, arrival, admission and therapy timing, and RTS. All these elements are important to identify the pattern and weakness in each trauma systems ${ }^{22,23,24}$. Our database will need more depth and elements modification to make it more complete.

One of the major problems we encountered during data collection was missing and incomplete data. Because of this problem, we had to extract data only from general surgery, emergency and medical records. Pure neurosurgery and orthopaedic trauma cases had to be excluded due to the large number of missing and incomplete data. We also noted the problem in handling and computing data which is prone to error and repetitions when keyed in manually by specialists, residents and junior staff. This may be due to the miscommunications and limited time.

Our trauma database is a modest effort with an aim to identify the pattern of trauma cases using the available data to support our intention in developing a dedicated trauma service. Having said that, a more thorough and systematic approach should be taken to improve the database. This include optimizing data collection method, training of a dedicated personnel, having a better data capturing software, involvement of appropriate authority and ensuring flow of funding. Although there is no standard software or parameters list but most registry include the basic demographics, ward related information, AIS and any population specific information from their epidemiology studies.
We have identified few limitations of this study. Firstly, this is a retrospective data in a single tertiary university hospital. Secondly, exclusion of missing and incomplete data might cause inaccurate representation within the trauma scoring. The exclusion of pure neurosurgery and orthopaedic cases also might conceal the true amount of trauma cases presented to our institution. Thirdly, the wide variation of certain parameters such as demographics and injuries type cause inaccurate analysis hence the data may not be representative of a true cause of morbidity and mortality. Finally, we do acknowledge a possibility of more data loss due to lack of software or dedicated personnel for data handling and capturing.

\section{CONCLUSION}

The available data clearly suggests the need for a dedicated trauma services or at least a more stringent trauma protocol which encompasses all departments. Steps should be taken to modify this data compilation and organization to ensure more quality and reliable data collection.

\section{CONFLICT OF INTEREST}

The authors declare that they have no competing interests in publishing this article.

\section{REFERENCES}

1. Global status report on road safety: Time for action. (2009). Geneva, World Health Organization.

2. American College of Surgeons. (2014). Resources for optimal care of the injured patient, 6th edition.

3. Royal Australian College of Surgeons. (2014). Guidelines for a structure approach to the provision of optimal trauma care.

4. NHS Clinical Advisory Groups Report: Regional Networks for Major Trauma (2010): 371 - 378. Available from: http:// www.uhs.nhs.uk/Media/SUHTInternet/ Services/Emergencymedicine / Regionalnetworksformajortrauma.pdf 
5. Gabbe BJ, Simpson PM, Sutherland AM et al. (2002). Improved functional outcomes for major trauma patients in a regionalized, inclusive trauma system. Ann Surg 255: 1009 $-1015$.

6. Baker SP, O'Neill B, Haddon W Jr, Long WB. (1976). J Trauma 14: 187 - 196.

7. Association of the Advancement of Automotive Medicine. (2015). Abbreviated Injury Scale (AIS). Retrieveed from www. aamm.org/abbreviated-injury-scale-ais

8. Morrissey BE, Delaney RA, Johnstone AJ et al. (2015). Do trauma systems work? A comparison of major trauma outcomes between Aberdeen Royal Infirmary and Massachusetts General Hospital. Injury 46: $150-155$.

9. MacKenzie EJ, Rivara FP, Jurkovich GJ et al. (2006). A national evaluation of the effect of trauma-center care on mortality. $\mathrm{N}$ Engl J Med 354: 366 - 378.

10. Egberink RE, Otten HJ, IJzerman MJ et al. (2015). Trauma team activation varies across Dutch emergency departments: a national survey. Scandinavian Journal of Trauma, Resuscitation and Emergency Medicine 23: 100.

11. Bukur M, Habib F, Catino J et al. (2015). Does unit designation matter? A dedicated trauma intensive care unit is associated with lower post injury complication rates and death after major complication. J Trauma Acute Care Surg 78: 920 - 927.

12. Davenport RA, Tai N, West A et al. (2010). A major trauma centre is a specialty hospital not a hospital of specialties. Br J Surg 97: $109-117$.

13. Ursic C, Curtis K, Zou Y, Black D. (2009). Improved trauma patient outcomes after implementation of a dedicated trauma admitting service. Injury 40: 99 - 103.
14. Beuran M, Stoica B, Negoi I et al. (2014). Trauma registry - A necessity of modern clinical practice. Chirurgia 109: 157 - 160.

15. Cameron PA, Gabbe BJ, Cooper DJ et al. (2008). A statewide system of trauma care in Victoria: effect on patient survival. Med J Aust 189: 546 - 550 .

16. Shaban S, Eid HO, Barka E, Abu-Zidan FM. (2010). Towards a national trauma registry for the United Arab Emirates. BMC Research Notes 3: 187.

17. Mehmood A, Razzak JA, Kabir S et al. (2013). Development and Pilot Implementation of a Locally Developed Trauma Registry: Lessons Learnt in a Low-Income Country. BMC Emerg Med 13: 4.

18. Hasler RM, Srivastava D, Aghayev E et al. (2014). First results from a Swiss level I trauma centre participating in the UK Trauma Audit and Research Network (TARN): Prospective cohort study. Swiss Med Wkly 144: w13910.

19. Njihia BN, Saidi H, Ogeng'o JA. (2016). Preliminary Data from a De Novo Trauma Registry. The Annals of African Surgery 13: 1.

20. Stoica B, Paun S, Tanase I et al. (2015). Trauma pattern in a level 1 east-European trauma center. Journal of Acute Disease 4: $322-326$.

21. Byun CS, Park IH, Oh JH et al. (2015). Epidemiology of trauma patients and analysis of 268 mortality cases: Trends of a single center in Korea. Yonsei Medical Journal 56: 220 - 226.

22. Gruen RL, Gabbe BJ, Stelfox HT, Cameron PA. (2012). Indicators of the quality of trauma care and the performance of trauma systems. British Journal of Surgery 99: 97 104.

23. American College of Surgeons Committee on Trauma. (2004). National Trauma Data Bank Report 2004. Chicago: American College of Surgeons.

24. Brinck T, Handolin L, Paffrath $T$, Lefering T. (2015). Trauma registry comparison: Six-year results in trauma care in Southern Finland and Germany. Eur J Trauma Emerg Surg 41: $509-516$. 
